\title{
microRNA-143, down-regulated in osteosarcoma, promotes apoptosis and suppresses tumorigenicity by targeting Bcl-2
}

\author{
HAO ZHANG ${ }^{*}$, XIAOBING CAI*, YANG WANG, HAO TANG, DAKI TONG and FANG JI
}

Department of Orthopedics, Changhai Hospital, Second Military Medical University, Shanghai 200433, P.R. China

Received June 18, 2010; Accepted August 3, 2010

DOI: $10.3892 /$ or_00000994

\begin{abstract}
Deregulated microRNAs and their roles in tumorigenesis are still largely unknown. Here, we focused on the roles of miR-143 in osteosarcoma, as previous reports have suggested its importance in some other types of cancer. We found that miR-143 was down-regulated in osteosarcoma cell lines and primary tumor samples, and the restoration of miR143 reduced cell viability, promoted cell apoptosis and suppressed tumorigenicity. Additionally, Bcl-2, an important antiapoptotic molecule, was identified to be a novel direct target of miR-143, and the proapoptotic function of miR-143 is further suggested to be mainly through the targeting of Bcl-2 expression. Collectively, our data identify the important roles of miR-143 in osteosarcoma pathogenesis and indicate its potential application in cancer therapy.
\end{abstract}

\section{Introduction}

microRNAs (miRNAs) are an abundant class of 17-25 nucleotide small non-coding RNAs. They regulate gene expression at the post-transcriptional level through binding to the $3^{\prime}$ untranslational region ( 3 'UTR) of target mRNAs. Since the initial observation, 1000 miRNAs have been identified in mammals, however, biological functions of a large part remain illusive. Growing evidence has suggested that miRNAs play important roles in the regulation of diverse biological processes (1), and their deregulation or dysfunction partici-pate in cancer development and clinical outcomes of cancer patients (2). However, deregulated microRNAs and their roles in tumorigenesis are still largely unknown.

Osteosarcoma is the third most common cancer in childhood and young adults and the most common cancer of bone characterized by an aggressive clinical course (3). For patients having no metastatic disease at diagnosis, the 5-year survival is $60-70 \%$ (4). However, for patients who present

Correspondence to: Professor Fang Ji, Department of Orthopedics, Changhai Hospital, Second Military Medical University, 800 Xiangyin Road, Shanghai 200433, P.R. China

E-mail: doctorjif@yahoo.com.cn

*Contributed equally

Key words: microRNA, miR-143, apoptosis, Bcl-2, osteosarcoma with metastatic disease or whose tumor recurs, the clinical outcomes are far worse (5). The mechanisms that orchestrate the multiple oncogenic insults required for initiation and progression of osteosarcoma are not clear. To date, miRNAs have been suggested to participate in osteosarcoma development, such as miR-31 (6), miR-34 (7) and miR-140 (8). However, as only a few miRNAs were reported to be involved in osteosarcoma, we are still at the beginning of revealing the roles of deregulated miRNAs in osteosarcoma carcinogenesis and progression.

Recently, miR-143 has been reported to be downregulated in some types of cancer, such as colorectal cancer (9-13), prostate cancer $(14,15)$, oral squamous cell carcinoma (16), pituitary tumor (17), cervical cancer (18), nasopharyngeal carcinoma (19) and lymphoma $(20,21)$. In these malignancies, forced expression of miR-143 inhibits cancer cell growth. However, whether miR-143 is deregulated in osteosarcoma and its roles in osteosarcoma carcinogenesis and progression are still elusive.

In the present study, we found that miR-143 was downregulated in osteosarcoma cell lines and primary tumor samples, and miR-143 was further identified to be a tumor suppressor, as restoration of miR-143 expression in osteosarcoma cell lines was able to reduce cell viability and promote cell apoptosis in vitro, and suppress tumorigenicity in vivo. Additionally, Bcl-2, an important antiapoptotic molecule, was identified to be a novel direct target of miR143 , and the proapoptotic function of miR-143 is further suggested to be mainly through targeting Bcl-2 expression. Thus, our data suggest important roles of miR-143 in osteosarcoma pathogenesis and indicate its potential application in cancer therapy.

\section{Materials and methods}

Patients and tumor samples. Surgically resected paired osteosarcoma tumor tissues and adjacent normal tissues used for qRT-PCR and Western blot analysis were collected from 30 primary osteosarcoma patients during operation between 2006 and 2009 at Changhai Hospital (Shanghai, China), as detailed in Table I. Surgically removed tissues were quickly frozen in liquid nitrogen until analysis. All samples were collected with the informed consent of the patients and the experiments were approved by the ethics committee of Second Military Medical University, Shanghai, China. The investigations were conducted according to the Declaration of Helsinki principles. 
Table I. Characteristics of osteosarcoma patients.

\begin{tabular}{|c|c|c|c|c|c|c|c|}
\hline No. & Sex & Age & Locus & Source & Stage & M. & $\mathrm{AKP}(\mathrm{U} / \mathrm{l})$ \\
\hline 1 & M & 15 & Femur & Primary OS & II B & - & $934(\mathrm{H})$ \\
\hline 2 & M & 16 & Femur & Primary OS & II B & - & $132(\mathrm{H})$ \\
\hline 3 & M & 13 & Femur & Primary OS & II B & - & 83 \\
\hline 4 & $\mathrm{~F}$ & 18 & Tibia & Primary OS & II B & - & 92 \\
\hline 5 & M & 24 & Femur & Primary OS & II B & - & $383(\mathrm{H})$ \\
\hline 6 & M & 53 & Femur & Primary OS & II B & - & $149(\mathrm{H})$ \\
\hline 7 & M & 71 & Humerus & Primary OS & II B & - & $140(\mathrm{H})$ \\
\hline 8 & M & 20 & Femur & Primary OS & II B & - & $435(\mathrm{H})$ \\
\hline 9 & M & 53 & Costa & Primary OS & II A & - & 98 \\
\hline 10 & $\mathrm{~F}$ & 17 & Femur & Primary OS & II B & - & 59 \\
\hline 11 & M & 31 & Femur & Primary OS & II B & - & $112(\mathrm{H})$ \\
\hline 12 & M & 32 & Femur & Primary OS & II B & - & $236(\mathrm{H})$ \\
\hline 13 & M & 11 & Tibia & Primary OS & II B & - & $431(\mathrm{H})$ \\
\hline 14 & M & 20 & Femur & Primary OS & II B & - & $125(\mathrm{H})$ \\
\hline 15 & M & 15 & Fibula & Primary OS & II B & - & $319(\mathrm{H})$ \\
\hline 16 & $\mathrm{~F}$ & 25 & Femur & Primary OS & II B & - & $129(\mathrm{H})$ \\
\hline 17 & M & 16 & Tibia & Primary OS & II B & - & $342(\mathrm{H})$ \\
\hline 18 & M & 17 & Femur & Primary OS & II B & - & $35(\mathrm{H})$ \\
\hline 19 & $\mathrm{~F}$ & 17 & Femur & Primary OS & II B & - & 89 \\
\hline 20 & $\mathrm{~F}$ & 38 & Fibula & Primary OS & II B & - & 46 \\
\hline 21 & $\mathrm{~F}$ & 46 & Calcaneus & Primary OS & II B & - & 97 \\
\hline 22 & $\mathrm{~F}$ & 16 & Femur & Primary OS & II B & - & $325(\mathrm{H})$ \\
\hline 23 & M & 17 & Ilium & Primary OS & II B & - & $131(\mathrm{H})$ \\
\hline 24 & M & 20 & Tibia & Primary OS & II B & - & 96 \\
\hline 25 & $\mathrm{~F}$ & 19 & Tibia & Primary OS & II B & - & $372(\mathrm{H})$ \\
\hline 26 & $\mathrm{~F}$ & 68 & Vertebrae & Primary OS & II B & - & 71 \\
\hline 27 & $\mathrm{~F}$ & 16 & Femur & Primary OS & II A & - & 88 \\
\hline 28 & M & 13 & Fibula & Primary OS & II B & - & $316(\mathrm{H})$ \\
\hline 29 & M & 15 & Femur & Primary OS & II B & - & $642(\mathrm{H})$ \\
\hline 30 & M & 23 & Femur & Primary OS & II B & - & 88 \\
\hline
\end{tabular}

M, male; F, female; OS, osteosarcoma; M., metastasis; AKP, alkaline phosphatase; H, high.

Cell culture. Human normal osteoblastic cell line hFOB 1.19, human osteosarcoma cell lines MG63 and U2OS, and human model cell line HEK293 were obtained from American Type Culture Collection (ATCC) and cultured in DMEM (Invitrogen) supplemented with heat-inactivated $10 \%$ fetal bovine serum (Invitrogen) at $37^{\circ} \mathrm{C}$ in a humidified incubator containing $5 \% \mathrm{CO}_{2}$. Bcl-2 stably overexpressed MG63 cells were transfected and selected in $800 \mu \mathrm{g} / \mathrm{ml} \mathrm{G} 418$ for 3-4 weeks and then confirmed by Western blot analysis for the expression of Bcl-2.

$R N A$ extraction and $q R T-P C R$. Total RNA, including miRNA, was extracted using miRNeasy kit (Qiagen) according to the manufacturer's instructions. To detect miRNA expression, real-time quantitative RT-PCR (qRT-PCR) analysis was performed using LightCycler (Roche) and SYBR RT-PCR kit (Takara). For miRNA analysis, stem-loop RT primer for miR-143 was 5'-GTCGTATCCAGTGCAGGGTCCGAG GTATTCGCACTGGATACGACGAGCTA-3', and q-PCR primers were 5'-AGTCAGTGAGATGAAGCACTG-3' (forward) and 5'-GTGCAGGGTCCGAGGT-3' (reverse) (22). Similarly, internal control U6 small nuclear RNA was quantified using its reverse primer for RT reaction and its forward and reverse primers for q-PCR, which was 5'-CTCG CTTCGGCAGCACA-3' (forward) and 5'-AACGCTTCACG AATTTGCGT-3' (reverse). The relative expression level of miR-143 was normalized to that of internal control U6 by using $2^{-\Delta \Delta C t}$ cycle threshold method $(23,24)$.

Transfection. MG63 $\left(5 \times 10^{3}\right)$ or U2OS $\left(5 \times 10^{5}\right)$ cells were seeded into each well of 96- or 6-well plate, respectively and incubated overnight, then transfected with negative control (NC) RNA or miR-143 mimics, synthesized by GenePharma, using Interfer in transfection reagent (Polyplus-transfection) at a final concentration of $50 \mathrm{nM}$. The NC RNA sequence was 5'-UUCUCCGAACGUGUCACGUTT-3' (sense) and 5'-ACGUGACACGUUCGGAGAATT-3' (antisense).

Analysis of cell viability in vitro. The in vitro cell viability of MG63 or U2OS cells transfected with NC or miR-143 was 
A

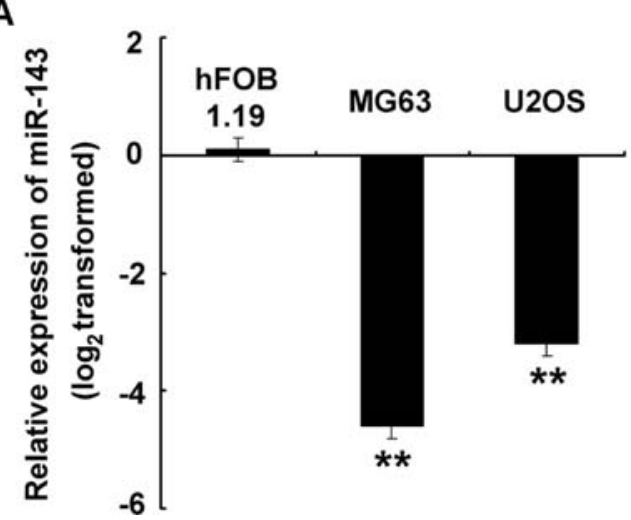

B

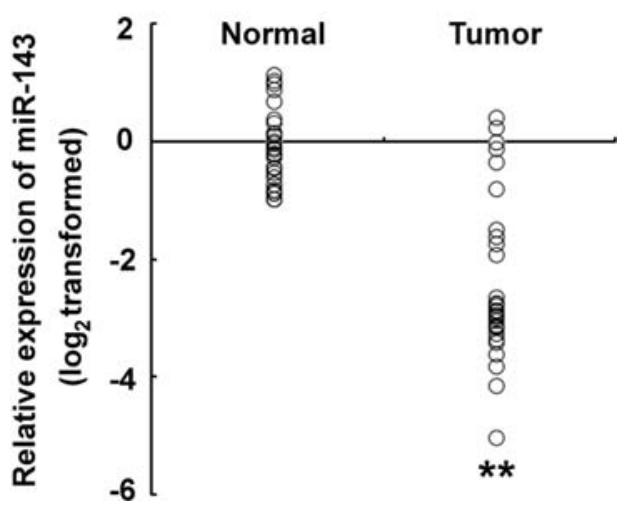

Figure 1. miR-143 is down-regulated in osteosarcoma cell lines and primary tumor samples. Expression of miR-143 was detected in human normal osteoblastic hFOB 1.19 cell line vs. human osteosarcoma MG63 and U2OS cell lines (A) and 30 matched human primary adjacent normal tissues vs. osteosarcoma tumor tissues (B) using qRT-PCR. Expression of miR-143 was normalized to that of U6 in each sample. Data are shown as mean \pm SD (n=6) in cell lines $(\mathrm{A})$ or displayed separately in human primary samples $(\mathrm{B}) .{ }^{* *}$ Significant differences $(\mathrm{P}<0.01)$.

measured using the MTT method (25). In brief, cells were seeded into 96-well plates and transfected. In the indicated time periods, $0.1 \mathrm{ml}$ of spent medium was replaced with an equal volume of fresh medium containing MTT $0.5 \mathrm{mg} / \mathrm{ml}$. Plates were incubated at $37^{\circ} \mathrm{C}$ for $4 \mathrm{~h}$, then the medium was replaced by $0.1 \mathrm{ml}$ of DMSO (Sigma) and plates shaken at room temperature for $10 \mathrm{~min}$. The absorbance was measured at $570 \mathrm{~nm}$.

Detection of apoptosis. Osteosarcoma MG63 or U2OS cells were transfected with $\mathrm{NC}$ or miR-143 respectively. At $48 \mathrm{~h}$ post-transfection, spent cell culture medium was replaced with serum-free DMEM. In the indicated time periods post serum deprivation, cells were harvested, washed, resuspended in the staining buffer, and examined with Vybrant Apoptosis Assay kit (Invitrogen). Stained cells were detected by FACSCalibur and data were analyzed with CellQuest software (both from Becton-Dickinson). The Annexin V-positive and propidium iodide (PI)-negative cells were regarded as apoptotic cells.

Tumorigenicity assay in nude mice. All experiments involving animals were undertaken in accordance with the National Institute of Health Guide for the Care and Use of Laboratory Animals, with the approval of the Scientific Investigation Board of Second Military Medical University, Shanghai. The tumorigenicity assay was performed as reported $(26,27)$. In detail, NC or miR-143 transfected osteosarcoma MG63 or U2OS cells $\left(1 \times 10^{6}\right)$ were suspended in $0.1 \mathrm{ml}$ PBS and then injected subcutaneously into either side of the posterior flank of the same female BALB/c athymic nude mice at 4 weeks of age. Tumor growth was measured using caliper daily, and tumor volume was calculated according to the formula: volume $=$ length $\mathrm{x}$ width ${ }^{2} \times 0.5$.

3'UTR luciferase reporter assay. The human B-cell CLL/ lymphoma 2 (Bcl-2) 3'UTR luciferase reporter construct was made by cloning human Bcl-2 mRNA 3'UTR sequence into the 3'UTR region of pMIR-Report construct (Promega). miR-143 target site deleted Bcl-2 3'UTR luciferase reporter construct was made by PCR fragments of Bcl-2 3'UTR luciferase reporter construct lacking the target site and ligated. MG63 cells $\left(5 \times 10^{3}\right)$ were seeded into each well of 96-well plate and incubated overnight, then co-transfected with 80 ng Bcl-2 3'UTR Firefly luciferase reporter plasmid, 40 ng internal control pRL-TK-Renilla-luciferase plasmid, and indicated RNAs (final concentration, $50 \mathrm{nM}$ ). After $24 \mathrm{~h}$, luciferase activities were measured using Dual-Luciferase Reporter Assay System (Promega) according to the manufacturer's instructions. Data were normalized by dividing Firefly luciferase activity with that of Renilla luciferase as reported (24).

Western blot analysis. Cells and grinded tissues were lysed using M-PER Protein Extraction Reagent (Pierce) supplemented with protease inhibitor cocktail (Calbiochem). Protein concentrations of the extracts were measured with BCA assay (Pierce) and equalized with the extraction reagent. Equal amount of the extracts were loaded and subjected to SDSPAGE, transferred onto nitrocellulose membranes, and then blotted as reported (24). Antibodies specific to human Bcl-2 (2876) and $\beta$-actin (4967), and horseradish peroxidase-coupled secondary antibodies were purchased from Cell Signaling Technology.

Statistical analysis. Data are presented as mean \pm SD. Statistical comparisons between experimental groups were analyzed by Student's t-test and a two-tailed $\mathrm{P}<0.01$ was taken to indicate statistical significance.

\section{Results}

miR-143 is down-regulated in osteosarcoma cell lines and primary tumor samples. In order to gain insight into the biological roles of miR-143 in human osteosarcoma development, we compared miR-143 expression in human normal osteoblastic cell line hFOB 1.19 and human osteosarcoma cell lines MG63 and U2OS by real-time qRT-PCR. Compared to normal hFOB 1.19 cells, miR-143 expression was significantly decreased in osteosarcoma MG63 and U2OS cells (Fig. 1A). Furthermore, we collected 30 pairs of primary osteosarcoma tumor samples and adjacent normal tissues (Table I). Expression of miR-143 is significantly suppressed in 24 of $30(80 \%)$ tumor samples compared to that in adjacent 

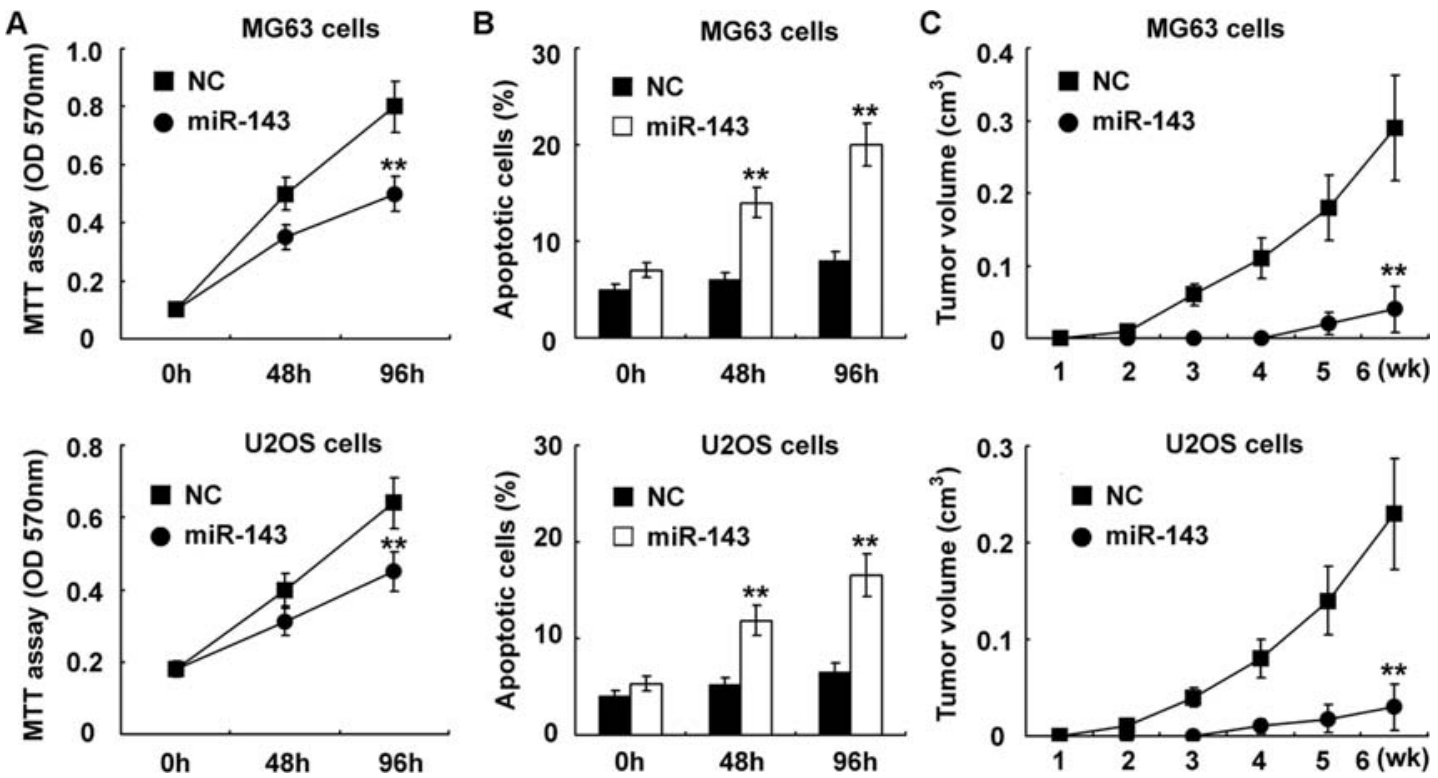

Figure 2. miR-143 reduces cell viability, promotes cell apoptosis and suppresses tumorigenicity. (A) Osteosarcoma MG63 or U2OS cells were transfected with $\mathrm{NC}$ or miR-143 mimics as indicated. Cell viability was measured in the indicated time periods post-transfection using MTT assay. (B) Osteosarcoma MG63 or U2OS cells were transfected as in (A). After $48 \mathrm{~h}$, cell culture medium was replaced with serum-free medium. Cell apoptosis was measured in the indicated time periods post serum deprivation. (C) Effect of miR-143 on tumorigenicity in nude mouse xenograft model. NC or miR-143 mimics transfected osteosarcoma MG63 or U2OS cells were injected subcutaneously into either side of the posterior flank of the same nude mice respectively. The tumor growth curve is shown as indicated. Data are shown as mean $\pm \mathrm{SD}(\mathrm{n}=6)$ of one representative experiment. Similar results were obtained in three independent experiments. ${ }^{* *}$ Significant differences $(\mathrm{P}<0.01)$.

A

Human Bcl-2 3'UTR (1811-1817)

5'-.......UUAAAAAAAUACAUGCAUCUCAG........3'

miR-143 3'-CUCGAUGUCACGAAG'ÜÁĠÁĠं-5'

Target site 2

Human Bcl-2 3'UTR (4513-4519)

5'- -.......CAAUCAUGAAAUAUGCAUCUCAC.......-3'

miR-143 3'-CUCGAUGUCACGAAG'ÜÁGÁĠं-5'

B

2 Empty vector
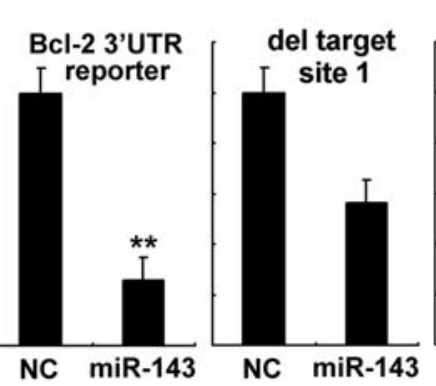

C
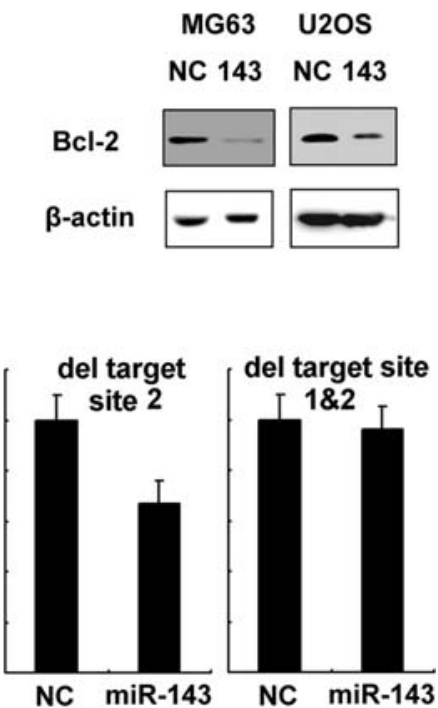

Figure 3. Antiapoptotic Bcl-2 is directly targeted and regulated by miR-143. (A) Human Bcl-2 might be molecular target of miR-143. Shown is a sequence alignment of miR-143 and its target sites in 3'UTR of Bcl-2, which was downloaded from TargetScan (http://www.targetscan.org). (B) MG63 cells were cotransfected with empty vector, human Bcl-2 3'UTR Firefly luciferase reporter plasmid, or the either one or both deletions of the putative target sites as indicated, together with pTK-Renilla-luciferase plasmids, NC or miR-143 mimics as indicated. After $24 \mathrm{~h}$, Firefly luciferase activity was measured and normalized by Renilla luciferase activity. (C) Osteosarcoma MG63 or U2OS cells were transfected as described in Fig. 2A. After 48 h, human Bcl-2 and $\beta$-actin were detected by Western blot analysis. Data are shown as mean \pm SD $(n=6)$. Similar results were obtained in three independent experiments. ${ }^{* *}$ Significant differences $(\mathrm{P}<0.01)$.

normal tissues (Fig. 1B). These results suggest that downregulation of miR-143 is, at least in part, involved in most of human osteosarcoma development.

miR-143 reduces cell viability, promotes cell apoptosis and suppresses tumorigenicity. The suppression of miR-143 prompted us to investigate whether miR-143 functions as a tumor suppressor in osteosarcoma. In MG63 and U2OS cells, restoration of miR-143 expression reduced cell viability in both of the osteosarcoma cell lines (Fig. 2A). Furthermore, miR-143 overexpression promoted cell apoptosis upon serum deprivation in osteosarcoma cells (Fig. 2B). These results 


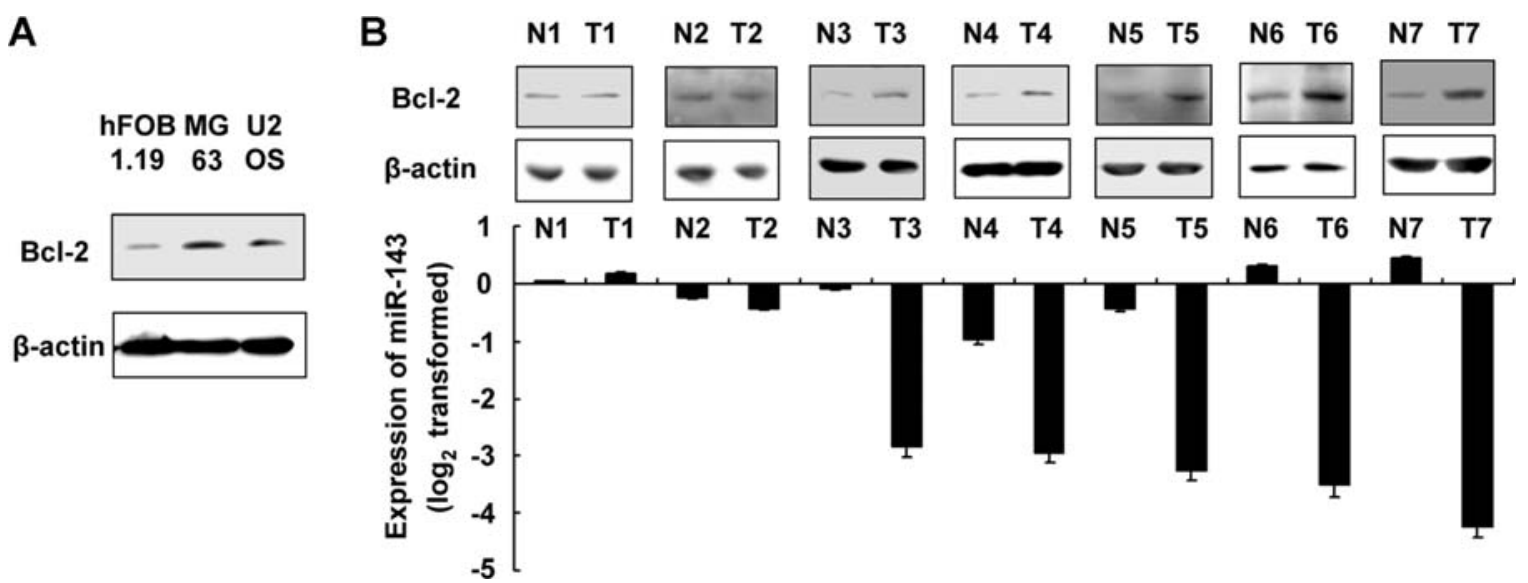

Figure 4. Bcl-2 expression is up-regulated in osteosarcoma. (A) Shown is the protein expression of Bcl-2 and B-actin in human normal osteoblastic hFOB 1.19 cell line versus human osteosarcoma MG63 and U2OS cell lines detected by Western blot analysis. (B) Expression of Bcl-2 and B-actin in available protein samples of matched human primary adjacent normal tissues versus osteosarcoma tumor tissues were detected by Western blot analysis. In these samples, expression of miR-143 was shown below.
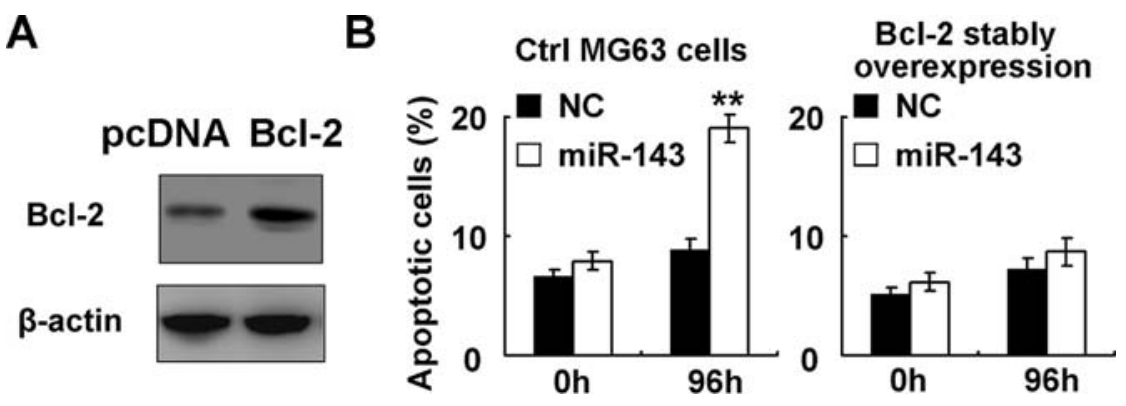

Figure 5. Proapoptotic function of miR-143 is mainly through targeting Bcl-2. (A) Bcl-2 expression in empty pcDNA vector or Bcl-2 stably overexpressed MG63 cells were detected by Western blot analysis. (B) MG63 cells in (A) were transfected as described in Fig. 2A. Cell apoptosis was measured 96 h post serum deprivation as in Fig. 2A. Data are shown as mean $\pm \mathrm{SD}(\mathrm{n}=6)$ of one representative experiment. Similar results were obtained in three independent experiments. ${ }^{* *}$ Significant differences $(\mathrm{P}<0.01)$.

demonstrate that miR-143 inhibits osteosarcoma growth in vitro.

Next, an in vivo model was subsequently applied to evaluate the effect of miR-143 on tumorigenicity. miR-143 transfected osteosarcoma MG63 and U2OS cells revealed a delayed tumor formation and a significant reduction in the tumor size compared to negative control transfectants (Fig. $2 \mathrm{C})$. This result further demonstrates the tumor suppressive effect of miR-143 on osteosarcoma.

Antiapoptotic Bcl-2 is a direct target of miR-143. We next sought to investigate the molecular mechanism responsible for the antitumor effect of miR-143 on osteosarcoma that was observed above. As miRNAs function mainly through inhibition of their target mRNAs via binding to the 3'UTR, we found that human Bcl-2, an important antiapoptotic molecule well-accepted to protect osteosarcoma cells from apoptosis $(28,29)$, contained two putative miR-143 target sites by TargetScan prediction (Fig. 3A). To verify whether Bcl-2 is a direct target of miR-143, a dual-luciferase reporter system was employed by co-transfection of miR-143 and a luciferase reporter plasmid containing 3'UTR of human Bcl-2 or bearing either one or both deletions of the putative target sites. We found that the luciferase activity was suppressed by miR-143 co-transfection, and deletion of either putative target sites partially decreased the suppression by miR-143 (Fig. 3B). Furthermore, deletion of both putative target sites in Bcl-2 3'UTR failed to be targeted by miR-143 cotransfection (Fig. 3B), further suggesting that Bcl-2 3'UTR is directly targeted by miR-143 expression. Additionally, in osteosarcoma MG63 and U2OS cells, endogenous expression of $\mathrm{Bcl}-2$ protein was suppressed by miR-143 transfection (Fig. 3C). These results demonstrate that endogenous Bcl-2 expression is directly targeted and regulated by miR-143 and suggest that miR-143 may exert its proapoptotic function via inhibiting Bcl-2 expression.

We further compared $\mathrm{Bcl}-2$ protein expression between human normal osteoblastic hFOB 1.19 cells and osteosarcoma MG63 and U2OS cells, as miR-143 was observed to be downregulated in osteosarcoma cells above. As shown in Fig. 4A, Bcl-2 protein expression was significantly up-regulated in osteosarcoma cells. Furthermore, in available protein samples of paired primary osteosarcoma tissues, we found that Bcl-2 protein expression was also up-regulated in miR-143 decreased osteosarcoma tissues (Fig. 4B). These results further confirm that endogenous Bcl-2 is regulated by miR-143 expression. 
Proapoptotic function of miR-143 is mainly through the targeting of $\mathrm{Bcl}-2$. As Bcl-2 is well-accepted to protect osteosarcoma cells from apoptosis $(28,29)$ and its expression is validated to be directly targeted by miR-143 above, we further investigated whether the proapoptotic function of miR-143 was mainly through targeting Bcl-2. We prepared Bcl-2 stably overexpressed cell clone of osteosarcoma MG63 cells which expressed Bcl-2 mRNA without its 3'UTR sequence. These cells had significantly elevated Bcl-2 protein expression (Fig. 5A). Since the Bcl-2 stably overexpressed MG63 cells transcribed Bcl-2 mRNA without its 3'UTR, miR-143 should no longer target Bcl-2 expression in these cells. As shown in Fig. 5B, miR-143 induced cell apoptosis was significantly decreased by Bcl-2 stable overexpression. These results confirm that the proapoptotic function of miR-143 is mediated by inhibiting its target the Bcl-2.

\section{Discussion}

Herein, we presented the down-regulation of miR-143 in osteosarcoma and suggested the antitumor effect of miR-143 in osteosarcoma pathogenesis. In combination with previous reports revealing the roles of miR-143 in some other types of cancer, we further confirmed that miR-143 may function as a tumor suppressor or an antionco-miR in cancer carcinogenesis and progression. However, underlying mechanisms that mediate the down-regulation of miR-143 in cancer is still elusive. As epigenetic alterations in cancer often mediate the deregulation of tumor suppressors or oncogenes (30), we will next focus on whether the down-regulation of miR-143 in osteosarcoma is mediated by epigenetic alterations, such as DNA methylation or histone modification. Moreover, we also noticed that miR-143 is not down-regulated in a small portion of osteosarcoma patients. Whether the prognosis of these patients is better or whether miR-143 expression is a predictor of prognosis are still important and interesting topics in the future.

The antitumor effect of miR-143 in osteosarcoma is validated both in vitro and in vivo, which is restoration of miR-143 expression significantly reduces cell viability, promotes cell apoptosis and suppresses tumorigenicity. As re-expression of miRNAs is believed to hold substantial clinical potential in cancer therapy (31), it is obviously suggested that re-expression of miR-143 may have considerable potential for clinical treatment of osteosarcoma patients, especially for those who respond poorly to chemotherapy.

Human important antiapoptotic Bcl-2 is identified to be a novel direct target of miR-143 in osteosarcoma, suggesting that miR-143 may exert its proapoptotic function via inhibiting Bcl-2 expression. Previous reports have shown that human DNMT3A, KRAS and ERK5 are also molecular targets of miR-143 and they all participate in cancer development (11-14). Our data suggest that the proapoptotic function of miR-143 in osteosarcoma cells is mainly through inhibiting its target Bcl-2. However, these results are consistent with current opinions that a single miRNA can target multiple mRNAs, named 'targetome', to post-transcriptionally regulate gene expression (32). Hence, it is probable that we are still far from unveiling the last target of miR-143, and some of these potential targets may be still unknown in osteosarcoma carcinogenesis and progression. According to this presumption, interesting future work is required to identify the targetome and entire roles of miR-143 in cancer development.

\section{Acknowledgements}

We thank Professor Zhengdong Cai, Dr Yun Yang and Dr Jia Meng for their helpful discussion. This project was supported by grants from the National Natural Science Foundation of China (30973019).

\section{References}

1. Bushati N and Cohen SM: microRNA functions. Annu Rev Cell Dev Biol 23: 175-205, 2007

2. Garzon R, Calin GA and Croce CM: MicroRNAs in cancer. Annu Rev Med 60: 167-179, 2009.

3. Longhi A, Errani C, De Paolis M, Mercuri M and Bacci G: Primary bone osteosarcoma in the pediatric age: state of the art. Cancer Treat Rev 32: 423-436, 2006.

4. Provisor AJ, Ettinger LJ, Nachman JB, et al: Treatment of nonmetastatic osteosarcoma of the extremity with preoperative and postoperative chemotherapy: a report from the Children's Cancer Group. J Clin Oncol 15: 76-84, 1997.

5. Ferguson WS and Goorin AM: Current treatment of osteosarcoma. Cancer Invest 19: 292-315, 2001.

6. Creighton CJ, Fountain MD, Yu Z, et al: Molecular profiling uncovers a p53-associated role for microRNA-31 in inhibiting the proliferation of serous ovarian carcinomas and other cancers. Cancer Res 70: 1906-1915, 2010.

7. He C, Xiong J, Xu X, et al: Functional elucidation of MiR-34 in osteosarcoma cells and primary tumor samples. Biochem Biophys Res Commun 388: 35-40, 2009.

8. Song B, Wang Y, Xi Y, et al: Mechanism of chemoresistance mediated by miR-140 in human osteosarcoma and colon cancer cells. Oncogene 28: 4065-4074, 2009.

9. Michael MZ, O'Connor SM, van Holst Pellekaan NG, Young GP and James RJ: Reduced accumulation of specific microRNAs in colorectal neoplasia. Mol Cancer Res 1: 882-891, 2003.

10. Borralho PM, Kren BT, Castro RE, Da Silva IB, Steer CJ and Rodrigues CM: MicroRNA-143 reduces viability and increases sensitivity to 5-fluorouracil in HCT116 human colorectal cancer cells. FEBS J 276: 6689-6700, 2009.

11. Ng EK, Tsang WP, Ng SS, et al: MicroRNA-143 targets DNA methyltransferases $3 \mathrm{~A}$ in colorectal cancer. Br J Cancer 101: 699-706, 2009.

12. Chen X, Guo X, Zhang H, et al: Role of miR-143 targeting KRAS in colorectal tumorigenesis. Oncogene 28: 1385-1392, 2009.

13. Motoyama K, Inoue H, Takatsuno Y, et al: Over- and underexpressed microRNAs in human colorectal cancer. Int J Oncol 34: 1069-1075, 2009.

14. Clapé C, Fritz V, Henriquet C, et al: miR-143 interferes with ERK5 signaling, and abrogates prostate cancer progression in mice. PLoS One 4: e7542, 2009.

15. Lee CY, Rennie PS and Jia WW: MicroRNA regulation of oncolytic herpes simplex virus-1 for selective killing of prostate cancer cells. Clin Cancer Res 15: 5126-5135, 2009.

16. Yu T, Wang XY, Gong RG, et al: The expression profile of microRNAs in a model of 7,12-dimethyl-benz(a)anthranceinduced oral carcinogenesis in Syrian hamster. J Exp Clin Cancer Res 28: 64, 2009.

17. Amaral FC, Torres N, Saggioro F, et al: MicroRNAs differentially expressed in ACTH-secreting pituitary tumors. J Clin Endocrinol Metab 94: 320-323, 2009.

18. Wang X, Tang S, Le SY, et al: Aberrant expression of oncogenic and tumor-suppressive microRNAs in cervical cancer is required for cancer cell growth. PLoS One 3: e2557, 2008.

19. Chen HC, Chen GH, Chen YH, et al: MicroRNA deregulation and pathway alterations in nasopharyngeal carcinoma. Br J Cancer 100: 1002-1011, 2009.

20. Akao Y, Nakagawa Y, Kitade Y, Kinoshita T and Naoe T: Downregulation of microRNAs-143 and -145 in B-cell malignancies. Cancer Sci 98: 1914-1920, 2007. 
21. Akao Y, Nakagawa Y, lio A and Naoe T: Role of microRNA143 in Fas-mediated apoptosis in human T-cell leukemia Jurkat cells. Leuk Res 33: 1530-1538, 2009.

22. Chen C, Ridzon DA, Broomer AJ, et al: Real-time quantification of microRNAs by stem-loop RT-PCR. Nucleic Acids Res 33: e179, 2005

23. Livak KJ and Schmittgen TD: Analysis of relative gene expression data using real-time quantitative PCR and the $2^{-\Delta \Delta C}$ method. Methods 25: 402-408, 2001

24. Hou J, Wang P, Lin L, et al: MicroRNA-146a feedback inhibits RIG-I-dependent Type I IFN production in macrophages by targeting TRAF6, IRAK1 and IRAK2. J Immunol 183: 2150-2158, 2009.

25. Alley MC, Scudiero DA, Monks A, et al: Feasibility of drug screening with panels of human tumor cell lines using a microculture tetrazolium assay. Cancer Res 48: 589-601, 1998.

26. Su H, Yang JR, Xu T, et al: MicroRNA-101, down-regulated in hepatocellular carcinoma, promotes apoptosis and suppresses tumorigenicity. Cancer Res 69: 1135-1142, 2009.
27. Xiong Y, Fang JH, Yun JP, et al: Effects of MicroRNA-29 on apoptosis, tumorigenicity, and prognosis of hepatocellular carcinoma. Hepatology 51: 836-845, 2009.

28. Nedelcu T, Kubista B, Koller A, et al: Livin and Bcl-2 expression in high-grade osteosarcoma. J Cancer Res Clin Oncol 134: 237-244, 2008

29. Zhao Y, Zhang CL, Zeng BF, Wu XS, Gao TT and Oda Y: Enhanced chemosensitivity of drug-resistant osteosarcoma cells by lentivirus-mediated Bcl-2 silencing. Biochem Biophys Res Commun 390: 642-647, 2009.

30. Esteller M: Epigenetics in cancer. N Engl J Med 358: 1148-1159, 2008.

31. Mishra PJ and Merlino G: MicroRNA reexpression as differentiation therapy in cancer. J Clin Invest 119: 2119-2123, 2009.

32. Selbach M, Schwanhäusser B, Thierfelder N, Fang Z, Khanin R and Rajewsky N: Widespread changes in protein synthesis induced by microRNAs. Nature 455: 58-63, 2008. 